\title{
Comparing Emission and Traffic Flow models of different categories
}

\author{
Michael Behrisch; Jakob Erdmann \\ Institute of Transport Systems \\ German Aerospace Center \\ Berlin, Germany \\ Michael.Behrisch@dlr.de
}

\begin{abstract}
Emission modelling is one of the key applications of traffic simulation because it allows for the detailed evaluation of ITS and other traffic measures before implementation. In order to assess the outcomes correctly it becomes necessary to compare the different emission and traffic models for their applicability to different scenarios. This paper compares two different traffic models and three different emission models of diverse origins in an urban and a highway scenario.
\end{abstract}

Keywords-Emission Modelling; Microscopic Traffic Simulation

\section{INTRODUCTION}

The evaluation of ITS measures before implementation usually involves the setup of a (traffic) simulation scenario to quantify both the immediate effects on the traffic situation (concerning local speeds and global travel times) but also secondary effects like pollution and fuel consumption. Since there is a variety of models and implementations available which differ considerably in their approaches the natural question arises whether those models give comparable results at least for standard scenarios. In this paper we compare two different traffic simulation approaches one microscopic based on the Krauß model [3] and one mesoscopic queuing model [5]. Both are evaluated using three different microscopic emission models derived from two macroscopic data sets based on the Handbook of Emission Factors HBEFA [7] and one submicroscopic model PHEM developed by the TU Graz. All comparisons are done using the SUMO traffic simulation package $[1,2]$ and focus on the values aggregated on edges or the whole network not on individual vehicles. The two traffic models serve as representatives for the classes of detailed microscopic simulations versus coarse queue modelling and can give an idea which level of detail is needed for emission modelling. The different emission models again serve as representatives for two different approaches one macroscopic (coming from the street level or even categories like HBEFA) and one microscopic (detailed vehicle modelling) as in PHEM.

The paper is organized as follows: After giving a brief account of prior work on the topic we shortly describe the models we compare and how they were derived. Afterwards there is a section on the traffic scenarios used and how the evaluations were performed. Then the results comparing traffic measures as well as emission values are presented and we close with an outlook to further research questions.

\section{A. Prior work and state of the art}

Traffic simulation and especially car following models have a history going back at least to the Gipps model [8] which tried to give simplistic formulas to derive complex traffic behavior. Comparisons are mostly done on a microscopic basis comparing individual vehicle trajectories or on an aggregated level using traffic flows and speeds, see for instance [9] and the references therein.

The topic of emission and fuel consumption modelling arose in the last two decades with the increasing concern about the increasing air pollution induced by individual traffic. The models are still in heavy development and give very different results, see [11] for a meta analysis. Some basic comparison of model features which are useful in the traffic simulation area can be found in [10]. The topic is still a very active research area with new models still being developed.

\section{TRAFFIC AND EMISSION MODELS}

There are four different (traffic) model categories which are relevant for our research in this paper.

- Submicroscopic models: the internal state of a vehicle described by elements such as chosen gear and engine revolutions is known with subsecond resolution

- Microscopic models: the external state of every vehicle described by features such as speed and position is known with a fine time resolution (at most one second)

- Mesoscopic models: vehicles are still modelled in an individual way but their positions and speeds are known only in an aggregated way (because of queueing models etc.)

- Macroscopic models: Traffic is only modelled by numeric flows on edges 
2015 Models and Technologies for Intelligent Transportation Systems (MT-ITS)

3-5. June 2015. Budapest, Hungary

Those categories focus very much on the traffic side but describe also the values they can deliver as input to a potential emission model. Those values can be aggregated again such that it becomes feasible to apply an inherently submicroscopic model such as PHEM to a microscopic simulation as the default Krauß model in SUMO. This aggregation was done when implementing PHEMlight [6]. The disaggregation step (deriving a microscopic emission model from macroscopic data) is not as straightforward and is described in [6]. It resulted in two different emission models based on different versions of the HBEFA.

\section{SCEnARIOS AND Methodology}

In order to compare traffic modeling and emissions two different real world scenarios of comparable size but different traffic characteristics were used. The first is a part of the A92 a major motorway leading to the Munich airport. The track has a total length of about $25 \mathrm{~km}$ and was travelled by a total of 63171 vehicles $(11 \%$ trucks $)$ on the chosen day. The network was a manually polished version of a NavTeq network and the demand was calculated from induction loop measurements for July 1, 2013.

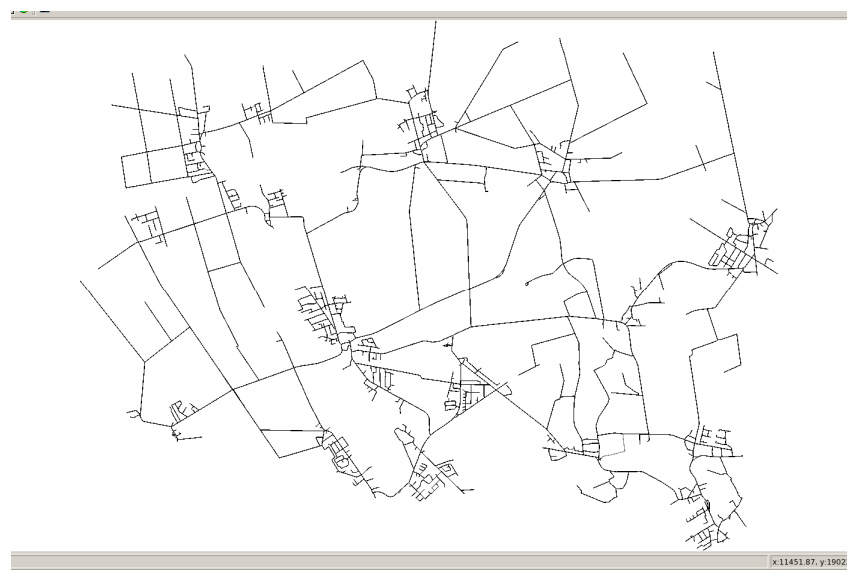

Fig. 1 The Brunswick network

The second network is a rural area in the northwest of the city of Brunswick (see Figure 1), including one traffic light. It was again based on a Navteq data set. The track has a total length of about $386 \mathrm{~km}$ (of which only $153 \mathrm{~km}$ were used by the traffic demand) and was travelled by a total of 41020 vehicles (3\% trucks) where the demand stems from the VALIDATE data set which gives origin destination matrices for the area around Brunswick. The route calculation involved a dynamic user assignment based on Gawron's algorithm.

Those two networks serve as representatives for an average motorway and an average rural scenario, both of comparable size and traffic volume. We decided to omit a city scenario for this study because of its higher complexity which needs a more detailed evaluation.

To evaluate the results we decided to focus on measurements which aggregate the data to edges and to examine the distribution of those edge errors. This gives on the one hand a rather global view on the average error per edge but allows on the other hand for further investigation about extremal edges as well. Furthermore SUMO facilitates the network wide visualization of such edge based values. The downside of this approach may be big differences in spatial aggregation because the edge lengths range from about $0.2 \mathrm{~m}$ to $2000 \mathrm{~m}$. Especially on very long edges local effects may be hidden in the aggregation.

For the traffic situation the average travel speed values were evaluated, which include the waiting times in front of traffic lights and before intersections but ignore travel (or stopping) times on the intersection. Additionally the travel times on the edges as well as the total number of samples were compared. Since all simulations ran with a step length of one second the samples correspond to the total number of seconds vehicles spent on the edge (if one vehicle spends five seconds on the edge and another ten, then a total of 15 samples is generated). This rather unusual value was chosen because it gives some kind of zeroth level approximation for the expected emissions, assuming a constant emission rate over time.

For the emissions we targeted mainly fuel consumption / $\mathrm{CO}_{2}$ (which have a linear relationship in all our emission models) and particular matter $\left(\mathrm{PM}_{\mathrm{x}}\right)$ and nitrogen oxides $\left(\mathrm{NO}_{\mathrm{x}}\right)$ emissions. All the used models can also calculate hydrocarbon (HC) and $\mathrm{CO}$ emissions which were omitted from this study because they offered no new insights into the general nature of the results.

All simulations were carried out using the most recent release 0.23 .0 of the SUMO simulation suite with the publicly available implementations of the HBEFA2, HBEFA3 and PHEMlight emission models. The emission classes used were in the HBEFA2 case the P_7_7 class for the passenger cars and the HDV_3_3 for the trucks, for HBEFA3 and PHEMlight the passenger cars were assumed to be Gasoline fueled while the trucks were Diesel fueled and both of Euro norm 5 which was considered to be the most established at least in Germany. We decided to stick to two emission classes only because the comparison should rather focus on the models (and the categories) itself and not be hidden by too much differentiation in the choice of emission classes. This limits of course the general applicability of the results since they apply strictly spoken only to the two emission classes evaluated.

The results presented in the following section give absolute and relative error values concerning the traffic related measurements (speed, time, samples) and total emission value sums in milligram comparing the two different simulation approaches and the three different models (so in general six plots) over the time scale of one day with the legend of Figure 2 , where the thick lines always stand for the microscopic model while the thin lines mean mesoscopic simulation. 
2015 Models and Technologies for Intelligent Transportation Systems (MT-ITS)

3-5. June 2015. Budapest, Hungary

\section{RESULTS AND COMPARISON}

\section{A. The highway scenario}

The highway scenario was driven at an average speed of $30.8 \mathrm{~m} / \mathrm{s}(110 \mathrm{~km} / \mathrm{h})$ which shows that the differences in travel speed between the microscopic and the mesoscopic shown in Figure 3 are relatively small

where the mesoscopic simulation tends to have higher speeds especially in the off-peak hours while in the peak hours



Fig. 2 Legend for the emission plots at least the maximum deviation shows that there are edges exhibiting a jammed state only in the mesoscopic simulation.
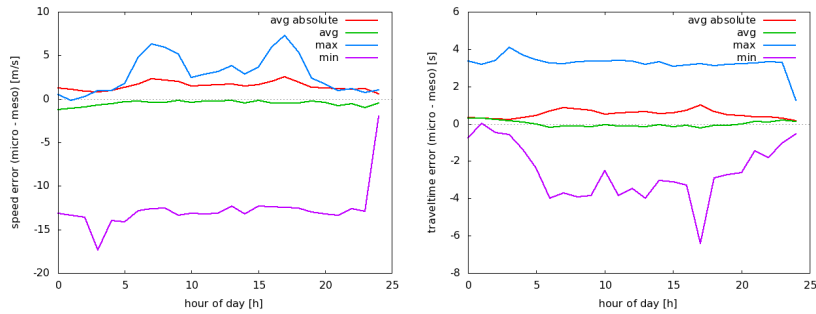

Fig. 3 Highway travel speed and travel time errors

The absolute travel time errors show less pronounced deviations which gives rise to the assumption that the speed deviations are concentrated rather on the short edges and do not contribute much to the big picture.

When looking at the sampled seconds in Figure 4 the average errors are still small while extremal cases during the rush hour are even better visible indicating that not only the speeds but also the number of vehicles differ considerably on those extremal edges.
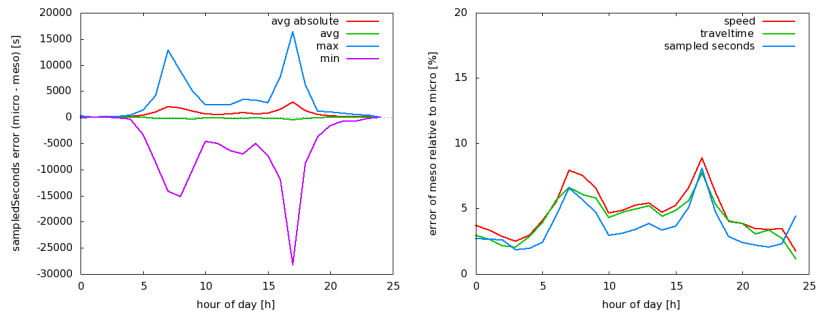

Fig. 4 Highway sample errors and relative errors in traffic measures

Overall (as shown in the second part of Figure 4) the relative errors in all traffic measures are well below $10 \%$ even in the peak hours leading to the result of a good agreement between the two models in the highway scenario.

Coming to the central point of emission modelling we change from error values to an absolute description because the deviations are very large in all cases. It seems to be a common "feature" of all emission models (see [11]) that predictions are only precise up to a small constant factor.

The $\mathrm{CO}_{2}$ emissions (which relate linearly to fuel consumption) shown in Figure 5 deviate considerably, especially the PHEMlight values seem to be very sensitive to the model used. One explanation would be that this model reacts heavily to accelerations which are not calculated in the mesoscopic model. Still the deviations are bound by a factor of 2 and more important show the same general shape.

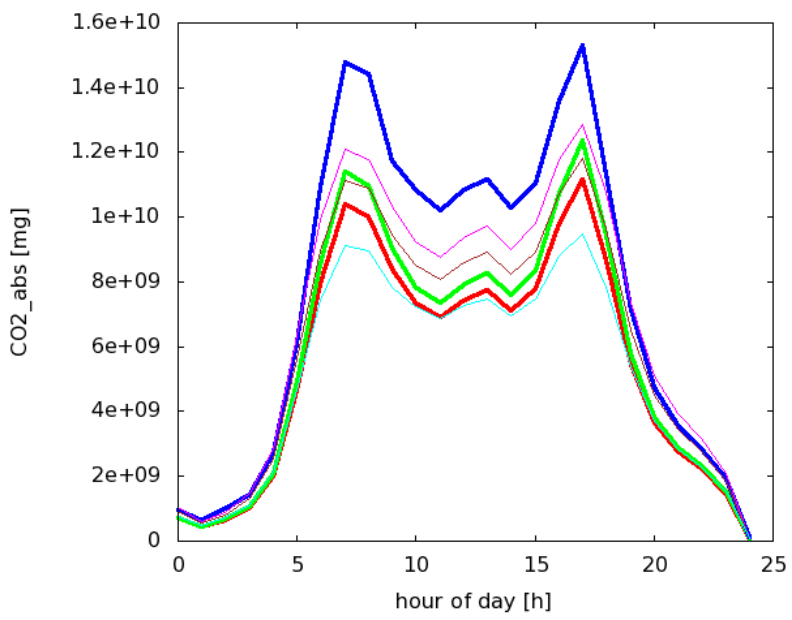

Fig. 5 Highway total $\mathrm{CO}_{2}$ emission

The two other emissions shown in Figure 6 differ even more but still differences between the emission models can be considerably larger than differences between the traffic models.
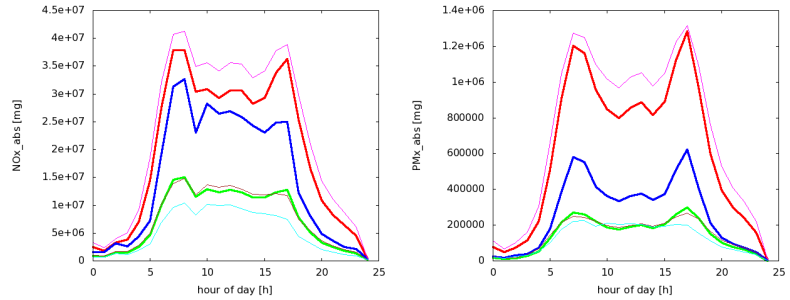

Fig. 6 Highway total $\mathrm{NO}_{\mathrm{x}}$ and $\mathrm{PM}_{\mathrm{x}}$ emission

\section{B. The rural scenario}

The rural scenario was driven at much smaller speeds of about $13 \mathrm{~m} / \mathrm{s}(46 \mathrm{~km} / \mathrm{h})$ but due to its higher junction dynamics the absolute errors in speed shown in Figure 7 have the same order as in the highway scenario. It is also interesting to note that there is a systematic bias (visible from the average error) in favor of the mesoscopic simulation which still needs to be evaluated.
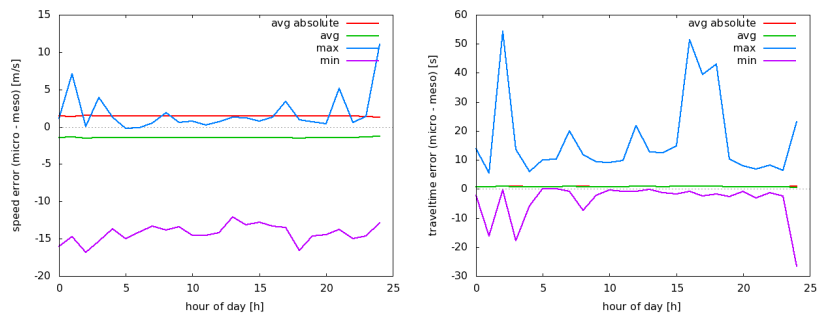

Fig. 7 Rural travel speed and travel time errors 
The travel time even shows some excessive outliers while being very close on average.

Those extreme outliers are still present in the samples plot in Figure 8 which shows that especially in the afternoon peak there are some edges which do not agree at all among both models. So while speed and travel time are still in the $10 \%$ error range the samples exceed this value by up to the $15 \%$ which leads to the expectation of higher differences in the emission values on those edges as well.
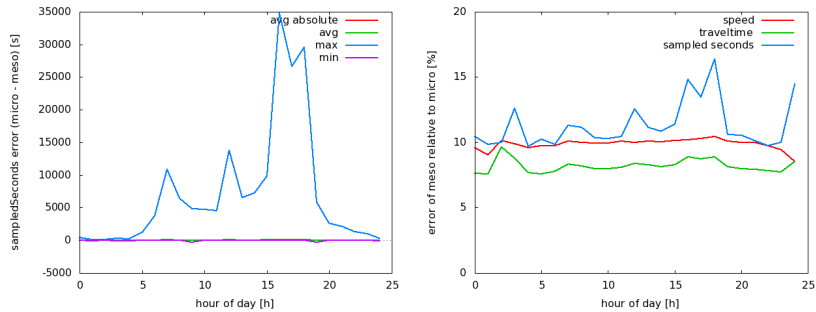

Fig. 8 Rural sample errors and relative errors in traffic measures

For the global $\mathrm{CO}_{2}$ emission picture in Figure 9 however the differences are still dominated by the difference between the different emission models, and the high volatility of the PHEMlight model which showed up in the highway scenario is present here again.

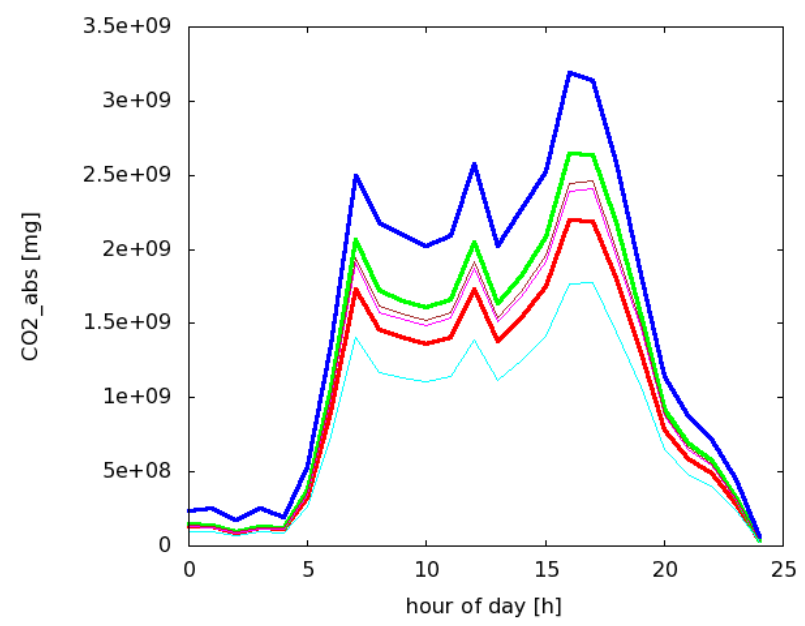

Fig. 9 Rural total $\mathrm{CO}_{2}$ emission

The most interesting part about the other emission values seems to be that although HBEFA2 again dominates the other models as in the highway case, the values seem to be in much better agreement between the two traffic models here. This is possibly due to the overall lower speeds.
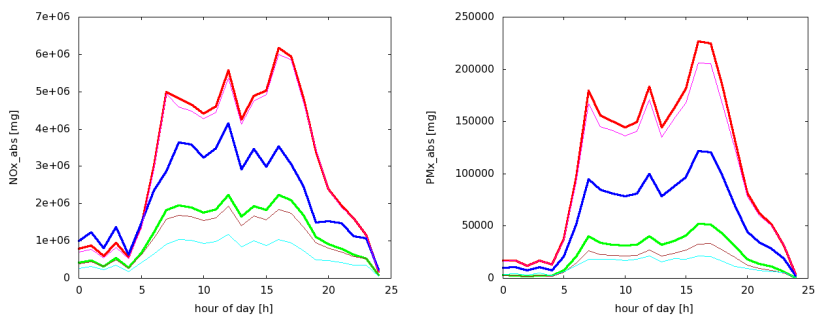

Fig. 10 Rural total $\mathrm{NO}_{\mathrm{x}}$ and $\mathrm{PM}_{\mathrm{x}}$ emission

\section{Summary}

We have compared traffic measures from a microscopic and a mesoscopic simulation model running the same traffic scenario. This was made possible by having both models integrated into the simulation software SUMO and being able to use identical input files.

Our first results show that the traffic data has significant local divergence between the simulation models but looks quite similar when aggregating over the whole simulation area. When comparing emission outputs, the differences between the emission models are on a similar scale as those between the simulation models. All results show a strong correlation over time and only differ in their scale when aggregating over the whole simulation area. For the emission models HBEFA2 and HBEFA3, $\mathrm{CO}_{2}$ emissions vary by less than $10 \%$ whereas PHEM shows a systematic divergence by as much as $100 \%$ between microscopic and mesoscopic simulation.

This supports our hypothesis that a substitution of the fast mesoscopic model for the more detailed microscopic model is feasible for some applications. However, when looking at the outliers, enormous discrepancies become visible. This suggests that there are some traffic situations in which the models diverge strongly. Preliminary investigations revealed the single traffic light in the overland scenario to be one source of such divergence. During the rush hour that respective intersection is saturated and small differences in regard to headways and driving-at-yellow are amplified. Additional investigations are necessary to either reduce these discrepancies through model improvements (without sacrificing speed) or to identify situations where model substitution is unwarranted.

Furthermore, we have compared different emission models which can be combined freely with the microscopic and mesoscopic traffic models. When looking at aggregated emissions for a whole scenario it is obvious that the differences between emissions models are generally larger than the differences between the traffic models. This is surprising under the assumption that emissions are strongly tied to vehicle accelerations since these accelerations are not modelled by the mesoscopic traffic model. Of the exemplary pollutant types, $\mathrm{CO}_{2}$ emissions have the lowest deviations (bounded by a factor of 2) whereas $\mathrm{NO}_{\mathrm{x}}$ and $\mathrm{PM}_{\mathrm{x}}$ show differences by a factor of up to 6 . Even then, the general shapes of the emission curves over time are strikingly similar. 
This suggests that either traffic and emission model may be useful to assess relative differences when comparing the impact of scenario changes (such as speed regulations or environmental zones) on emissions.

\section{OUTLOOK}

The aforementioned comparisons shall be extended to a city scenario which features dense traffic, a large number of traffic lights and (in the microscopic simulation) frequent lane changing. Due to the strong interactions between traffic participants and the much more chaotic nature of dense traffic, larger deviations in traffic measurements between the microscopic and mesoscopic simulations are to be expected. It will be interesting to find out whether mesoscopic emissions may nevertheless be used as a proxy for microscopic emissions. In order to support this assumption the evaluation should also be extended to more emission classes.

A second point of extension for this model comparison will be the look at a macroscopic emission model which can be supplied with aggregated traffic data from either microscopic or mesoscopic simulation as well as from a macroscopic traffic model.

The preliminary results show a good agreement between simulation models when aggregating data over the whole simulation area while still showing local divergence. This raises the question of representativeness when extrapolating our results to other simulation scenarios. A closer look at the root cause for divergence is therefore necessary.

\section{REFERENCES}

[1] D. Krajzewicz, J. Erdmann, M. Behrisch, L. Bieker, "Recent development and applications of SUMO - Simulation of Urban Mobility," in International Journal On Advances in Systems and Measurements, 5 (3\&4), 2012, pp. 128-138.

[2] SUMO - Simulation of Urban Mobility, available at http://sumo.dlr.de/

[3] S. Krauß (1998) Microscopic Modeling of Traffic Flow: Investigation of Collision Free Vehicle Dynamics. Dissertation. DLR-Forschungsbericht. $98-08,115 \mathrm{~S}$

[4] C. Gawron, "Simulation-Based Traffic Assignment"; $\mathrm{PhD}$ thesis, University of Cologne, Germany, 1998.

[5] N. Eissfeldt Vehicle-based modelling of traffic. Theory and application to environmental impact modelling. PhD thesis, Universität zu Köln, 2004.

[6] Krajzewicz, Daniel; Hausberger, Stefan; Wagner, Peter; Behrisch, Michael; Krumnow, Mario (2014) $2^{\text {nd }}$ Generation of Pollutant Emission Models for SUMO, SUMO2014 - $2^{\text {nd }}$ SUMO User Conference, Berlin, Germany

[7] Handbook of Emission Factors, available at http://www.hbefa.net/e/index.html

[8] P.G. Gipps, A behavioral car following model for computer simulation, Transp. Res. B 15, $105-111$ (1981).

[9] Brockfeld, Elmar und Kühne, Reinhart und Wagner, Peter (2004) Calibration and Validation of Microscopic Traffic Flow Models. In: TRB 2004 Annual Meeting, 1876 (TRB200), Seiten 62-70. TRB Annual Meeting, 2004-01-11 - 2004-01-15, Washington, DC (USA).

[10] iTETRIS Consortium, Deliverable D3.1: Traffic Modelling: Environmental Factors, February 2009, available via http://www.ictitetris.eu/

[11] Robin Smit, Leonidas Ntziachristos, Paul Boulter, Validation of road vehicle and traffic emission models - A review and meta-analysis, Atmospheric Environment, Volume 44, Issue 25, August 2010 